\title{
Adaptation to prismatic displacement as a function of the amount of available information
}

STANLEY COREN

STANFORD UNIVERSITY

The amount of information available during a pointing response while wearing displacing prisms was varied by allowing the arm to remain free or by constraining it to a track. There was significant adaptation in both conditions and the adaptation for the unconstrained or high information group was significantly greater.

The importance of knowledge of errors in adaptation to prismatic displacement has recently been in dispute. Held has maintained (Held \& Gotlieb, 1958; Held, 1961) that such information is unnecessary and offers as evidence the fact that his Ss adapt while their arms remain strapped to a pivoted paddle. With the arm thus constrained there is no opportunity for knowledge of response errors induced by the optical distortion.

Other investigators (Weinstein et al, 1964) contend that adaptation results from information gained about the nature of the distortion from knowledge of errors available in one's own responses. A recent study by Howard, Craske, \& Templeton (1965) seems to show that information about the nature of the distortion, even in the absence of a response, can produce adaptation to displacement.

To clarify the role of available information in adaptation, this study was undertaken.

\section{Method}

Twelve right handed students enrolled in an elementary psychology course participated as part of their course requirement. Each served under only one condition.

The apparatus was a table ( $5 \mathrm{ft} . \times 42$ in.) with a clear Plexiglas top and a removable cardboard cover. At one end of the table was a $U$ shaped notch for the S's head. A bite board served to hold S's head in place. A yardstick was attached to the underside of the table top, parallel to and 18 in. from $\mathrm{S}^{\dagger} \mathrm{s}$ frontal plane.

While in the apparatus, $S$ wore 25 diopter wedge prisms which displaced the target 4.5 in. The target, which was placed above the yardstick in the center of the table, was a wedge of wood with a darkened edge.

For adaptation session the cover was removed from the apparatus.

The high information group pointed 50 times to the target. They could clearly see any errors in localization.

The low information group had their right hand constrained on a track. The angle and inclination of the track had been determined from measurements taken on three pretest Ss, so that the motion of the hand along the track closely approximated the motion of the hand in freely pointing to the target. The track was mounted in the apparatus and the target placed at one end. S moved his hand up the track 50 times with the instruction "Move your hand up the track just as though you were pointing at the target."

With their hands constrained to the track the low information group could make no errors, hence they do not have the additional informational input available to the high information group who do have this feedback.

All measurements were taken with the table cover in place. This blocked S's view of his hand. Three premeasures with prisms in place were taken. Following adaptation three adaptation measures were taken with prisms in place and three aftereffect measures were taken with the naked eye.

Results

The results are summarized in Table 1.

The adaptation and the aftereffects are significant for both groups $(p<0.02)$. The difference between the high information and low information groups is significant at $p<0.02$ (Mann-Whitney $U$ test) for both the adaptation and the after effects.

\section{Discussion}

In the low information condition where the hand was constrained to the track and no information about the nature of the distortion is available from knowledge of response errors, there is still significant adaptation. This essentially verifies Held's contention that knowledge of errors is not necessary for adaptation. However the adaptation is approximately twice as large in the high information condition where this information is available, thus it appears that knowledge of errors can be used in adaptation.

A simple information processing model can not explain the adaptation in the low information condition, while a simple re-afference model cannot explain the increment in adaptation in the high information condition. These data suggest that both of these processes act simultaneously during the course of adaptation.

Table 1. Adaptation in inches

\begin{tabular}{lcc} 
& Adaptation & After effects \\
\hline High information & 3.27 & 2.37 \\
Low information & 1.52 & 1.27 \\
\hline
\end{tabular}




\section{References}

Held, R. Exposure history as a factor in maintaining stability of perception and coordination. J. nert. ment. Dis., 1961, 132 , 26-32.

Held, R., \& Gotlieb, N. Technique for studying adaptation to disarranged hand-eye coordination. Percept. mot. Skills, 1958 , 8. 83-86.
Howard, I. P., Craske, B., \& Templeton, W. B. Visumotor adaptation to discordant ex-afferent stimulation. J. exp. Psychol., $1965,70,189-191$.

Weinstein, S., Sersen, E. A., \& Weinstein, Deana. An attempt to replicate a study of disarranged eye-hand coordination. Percept. mot. Skills, 1964, 18, 629-632. 